\title{
Electrical and thermoelectrical properties of SnTe-based films and superlattices
}

\author{
Akihiro Ishida, ${ }^{1, a)}$ Tomohiro Yamada, ${ }^{1}$ Takuro Tsuchiya, ${ }^{1}$ Yoku Inoue, ${ }^{1}$ Sadao Takaoka, ${ }^{2}$ \\ and Takuji Kita ${ }^{3}$ \\ ${ }^{1}$ Department of Electrical and Electronic Engineering, Shizuoka University, Johoku 3-5-1, Naka-ku, \\ Hamamatsu 432-8561, Japan \\ ${ }^{2}$ Department of Physics, Graduate School of Science, Osaka University, Machikaneyama 1-1, \\ Toyonaka 560-0043, Japan \\ ${ }^{3}$ Toyota Motor Co., Mishuku 1200, Susono 410-1193, Japan
}

(Received 8 June 2009; accepted 30 August 2009; published online 23 September 2009)

\begin{abstract}
SnTe-based films and superlattices (SLs) were prepared and their electrical properties were measured. A EuTe/SnTe SL exhibited a hole mobility of $2720 \mathrm{~cm}^{2} / \mathrm{V}$ s, which is the highest value reported for any semiconductor material at room temperature. The SnEuTe film also exhibited high hole mobility in contrast to the PbEuTe system. These properties are explained in terms of the band offsets of EuTe/SnTe heterojunction and a decrease in the number of Sn vacancies. In addition, $\mathrm{SnTe} / \mathrm{PbSe}$ and $\mathrm{SnTe} / \mathrm{PbS}$ SLs with thin $\mathrm{SnTe}$ layers displayed $n$-type conduction with Seebeck coefficients comparable to those for $\mathrm{PbSe}$ and $\mathrm{PbS}$. These properties reflect the type II heterostructures. () 2009 American Institute of Physics. [doi:10.1063/1.3236541]
\end{abstract}

IV-VI compound semiconductors and quantum wells have a wide range of applications such as thermoelectric devices, infrared lasers, and detectors. Among these IV-VI materials, lead chalcogenides have been most extensively studied. On the other hand, SnTe-based materials have not received the attention owing to the high carrier concentration of SnTe. Although SnTe has a very small Seebeck coefficient, certain applications can be envisaged using quantum well systems which utilize the special band offsets with other materials such as $\mathrm{SnTe} / \mathrm{PbTe}$ system. We previously proposed that the $\mathrm{SnTe} / \mathrm{PbTe}$ heterojunction becomes type II where the valence band maxima of SnTe situate above the conduction band minima of $\mathrm{PbTe},{ }^{1}$ and explained the semimetallic properties of $\mathrm{SnTe} / \mathrm{PbTe}$ superlattices (SLs) in this manner. ${ }^{2,3}$ Rogacheva et al. ${ }^{4}$ reported the electrical and Seebeck properties of $\mathrm{SnTe} / \mathrm{PbTe} \mathrm{SLs}$, and attempted to explain their nonmonotonic dependence on the SnTe layer thickness by the coexistence of electrons and holes. These properties can be explained by the type II band offsets of the heterojunction. In this study, the electrical properties of EuTe/SnTe, $\mathrm{SnTe} / \mathrm{PbSe}$, and $\mathrm{SnTe} / \mathrm{PbS}$ SLs and SnEuTe films, including their Seebeck properties, are investigated and are discussed in terms of the band offsets at the heterojunctions.

Figure 1 shows the band diagram of a $\mathrm{SnTe} / \mathrm{PbTe} \mathrm{SL}$ proposed by Murase et al., ${ }^{1}$ including schematic subband structures and corresponding optical transitions. Real subband structures are more complicated owing to anisotropic effective masses of $L$-point valleys for PbTe and SnTe. Solid lines show conduction band subbands in the $\mathrm{PbTe}$ well, and dashed lines show valence band subbands in the SnTe well. In the $\mathrm{SnTe} / \mathrm{PbSe}$ and $\mathrm{SnTe} / \mathrm{PbS}$ SLs, similar band diagrams are expected. In the $\mathrm{SnTe} / \mathrm{PbTe}$ type II heterostructure, the valence band electrons in SnTe flow into the conduction band of PbTe. Thus, the SL becomes semimetallic, as ascertained by Hall measurements of SnTe/PbTe SLs. ${ }^{2,3}$ If the thickness of $\mathrm{SnTe}$ in the $\mathrm{SnTe} / \mathrm{PbTe} \mathrm{SL}$ is decreased, the

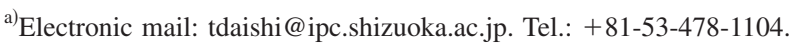

number of electrons flowing into the PbTe layer decreases, and the Fermi level approaches the bottom of the PbTe conduction band. Thus, $n$-type conduction similar to that in $n$-type $\mathrm{PbTe}$ should appear even if both the $\mathrm{PbTe}$ and the SnTe had $p$-type conduction. This phenomenon was reported by Rogacheva et al. ${ }^{4}$ for $n$-PbTe/p-SnTe SLs, and similar behavior should be expected for $\mathrm{SnTe} / \mathrm{PbSe}$ and $\mathrm{SnTe} / \mathrm{PbS}$ systems if similar type II heterostructures are formed. Figure 2 shows the band offsets expected for EuTe/PbTe and EuTe/ SnTe SLs. EuTe is a wide gap material with a band gap energy approximately $2 \mathrm{eV}$, and the EuTe/PbTe SL becomes type I, where the PbTe layers form quantum wells for both electrons and holes with comparable barrier heights. ${ }^{5,6}$ Considering the band offset of the $\mathrm{SnTe} / \mathrm{PbTe}$ system, it is expected that the barrier height in the conduction band would be much smaller in the EuTe/SnTe quantum well system, as shown in Fig. 2(b). In this study, the $\mathrm{SnTe} / \mathrm{PbSe}, \mathrm{SnTe} / \mathrm{PbS}$, and EuTe/SnTe, SLs were prepared by hot wall epitaxy. ${ }^{7}$ To prepare $\mathrm{SnTe} / \mathrm{PbSe}$ and $\mathrm{SnTe} / \mathrm{PbS}$ SLs, $5 \mathrm{~N}$ stoichiometric $\mathrm{SnTe}, \mathrm{PbSe}$, and $\mathrm{PbS}$ polycrystals were used as the source materials. Carrier types of the films prepared from the materials were $p$-type for $\mathrm{SnTe}$ and $\mathrm{PbSe}$, and $n$-type for $\mathrm{PbS}$. Carrier concentrations of $\mathrm{PbS}$ and $\mathrm{PbSe}$ were $\sim 10^{17} \mathrm{~cm}^{-3}$

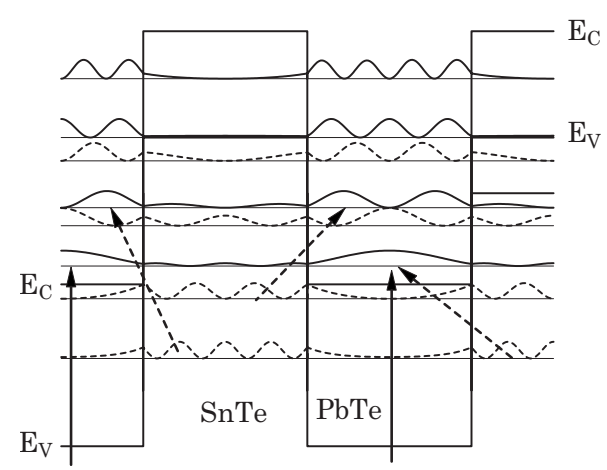

FIG. 1. Schematic band diagram of the SnTe/PbTe SL, subband structures, and possible optical transitions. 


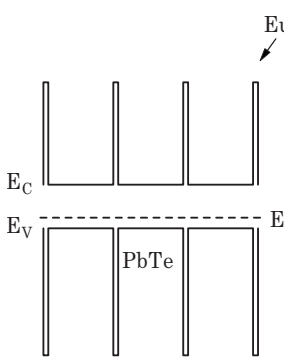

(a)

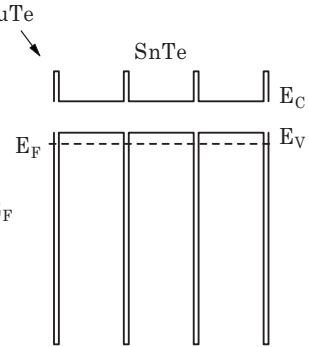

(b)
FIG. 2. Schematic energy band diagrams for EuTe/PbTe and EuTe/SnTe SLs.

and those of SnTe were $2 \times 10^{20} \mathrm{~cm}^{-3}$. To prepare the EuTe/ SnTe SL, 7-26 nm of SnTe and one atomic layer of Eu were deposited alternately to compensate for the strong stoichiometric deviation of the SnTe film. $\mathrm{Sn}_{1-x} \mathrm{Eu}_{x} \mathrm{Te}$ films with $x \approx 0.02$ were also prepared by simultaneous deposition of $\mathrm{SnTe}$ and Eu. Table I shows the electrical properties of these films and SLs at room temperature. Electrical properties of other IV-VI films and SLs are also shown for comparison.

The SnTe/PbSe SL with thin SnTe layer exhibited $n$-type conduction, even though both of SnTe and $\mathrm{PbSe}$ had $p$-type conduction, as expected from the type II band structure. The optical properties of this SL would be expected to be quite different to those of a semiconductor film. Figure 3 shows a comparison of the optical transmission spectra between the $\mathrm{PbSe}$ film and $\mathrm{SnTe} / \mathrm{PbSe} \mathrm{SL}$. Absorption coefficients, determined by simulations assuming an interband absorption and an exponential tail are shown as dashed-dotted lines, and the simulated spectra are shown by dashed line. ${ }^{6}$ The $\mathrm{SnTe} / \mathrm{PbSe}$ SL exhibited strong absorption above the band gap of $\mathrm{PbSe}$ $\left(2200 \mathrm{~cm}^{-1}\right)$. The exponential tail observed over the entire spectral range below the band gap of $\mathrm{PbSe}$ corresponds to electron transitions from the $\mathrm{SnTe}$ valence band to the $\mathrm{PbSe}$ conduction band as indicated by the dashed arrows in Fig. 1.

TABLE I. Electrical properties of $\mathrm{SnTe} / \mathrm{PbSe}, \mathrm{SnTe} / \mathrm{PbS}$, and EuTe/SnTe SLs and SnEuTe films at room temperature. Electrical properties of other IV-VI films and SLs are also shown for comparison.

\begin{tabular}{lccccc}
\hline \hline Sample & $\begin{array}{c}\text { Structure } \\
(\mathrm{nm} / \mathrm{nm})\end{array}$ & $\begin{array}{c}\mu \\
\left(\mathrm{cm}^{2} / \mathrm{Vs}\right)\end{array}$ & $\begin{array}{c}\text { Carrier con. } \\
\left(\mathrm{cm}^{-3}\right)\end{array}$ & Dopant & $\begin{array}{c}S \\
(\mu \mathrm{V} / \mathrm{K})\end{array}$ \\
\hline $\mathrm{SnTe}$ & & 203 & $p=2.0 \times 10^{20}$ & $\ldots$ & 6 \\
$\mathrm{PbSe}$ & & 534 & $p=3.5 \times 10^{17}$ & $\ldots$ & \\
$\mathrm{PbS}$ & & 491 & $n=7.8 \times 10^{17}$ & $\ldots$ & \\
$\mathrm{PbTe}$ & & 795 & $p=2.3 \times 10^{18}$ & $\mathrm{Tl}$ & \\
$\mathrm{PbTe}$ & & 1410 & $n=1.6 \times 10^{18}$ & $\mathrm{Bi}$ & \\
$\mathrm{SnTe} / \mathrm{PbSe}$ & $20 / 20$ & 107 & $p=1.9 \times 10^{19}$ & $\ldots$ & 43 \\
$\mathrm{SnTe} / \mathrm{PbSe}$ & $10 / 20$ & 249 & $n=5.1 \times 10^{18}$ & $\ldots$ & -103 \\
$\mathrm{SnTe} / \mathrm{PbSe}$ & $1.5 / 20$ & 783 & $n=1.5 \times 10^{18}$ & $\ldots$ & -254 \\
$\mathrm{SnTe} / \mathrm{PbS}$ & $1.5 / 25$ & 386 & $n=2.8 \times 10^{18}$ & $\ldots$ & -252 \\
$\mathrm{SnTe} / \mathrm{PbS}$ & $2 / 20$ & 283 & $n=2.3 \times 10^{18}$ & $\ldots$ & -266 \\
$\mathrm{SnTe} / \mathrm{PbS}$ & $2.5 / 30$ & 309 & $n=3.8 \times 10^{18}$ & $\ldots$ & -251 \\
$\mathrm{~Pb}{ }_{0.95} \mathrm{Eu} 0.05 \mathrm{Te}$ & & 49.7 & $p=1.1 \times 10^{18}$ & $\mathrm{Tl}$ & \\
$\mathrm{SnEuTe}$ & & 1740 & $p=1.8 \times 10^{19}$ & $\ldots$ & 27 \\
$\mathrm{SnEuTe}$ & & 2110 & $p=8.9 \times 10^{18}$ & $\ldots$ & 77 \\
$\mathrm{EuTe} / \mathrm{PbTe}$ & $0.3 / 10$ & 434 & $p=2.1 \times 10^{18}$ & $\mathrm{Tl}$ & \\
$\mathrm{EuTe} / \mathrm{SnTe}$ & $0.2 / 7$ & 1670 & $p=1.2 \times 10^{19}$ & $\ldots$ & 66 \\
$\mathrm{EuTe} / \mathrm{SnTe}$ & $0.3 / 14$ & 2080 & $p=1.1 \times 10^{19}$ & $\ldots$ & 63 \\
$\mathrm{EuTe} / \mathrm{SnTe}$ & $0.4 / 26$ & 2720 & $p=1.0 \times 10^{19}$ & $\ldots$ & 68 \\
\hline \hline
\end{tabular}

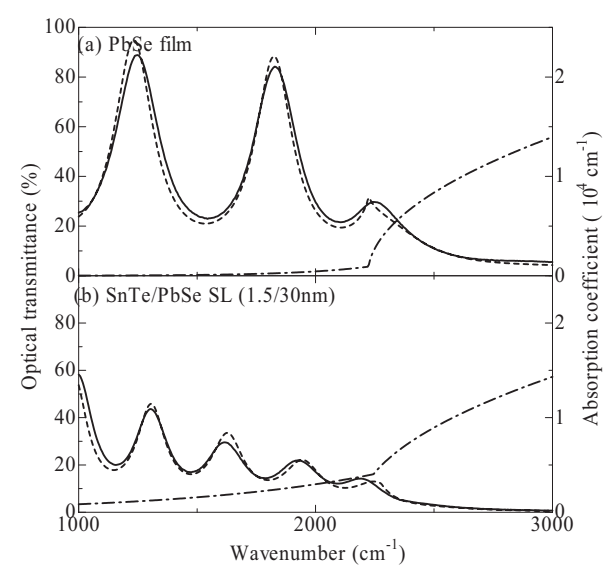

FIG. 3. Optical transmission properties of PbSe film and $\mathrm{SnTe} / \mathrm{PbSe} \mathrm{SL}$ with thickness of 1.6 and $3.1 \mu \mathrm{m}$, respectively. Solid and dashed lines show experimental and simulated transmission spectra, and dashed-dotted lines show corresponding absorption coefficients.

In the EuTe/SnTe SL, the hole concentration was greatly reduced compared to the value for $\mathrm{SnTe}$, and the room temperature hole mobility was drastically enhanced, as shown in Table I. A large enhancement in hole mobility was also observed for the SnEuTe ternary alloy films. Usually, the carrier concentration of $\mathrm{SnTe}$ decreases with increasing $\mathrm{Sn}$ content to $4 \times 10^{19} \mathrm{~cm}^{-3}$ with a hole mobility of about $900 \mathrm{~cm}^{2} / \mathrm{V} \mathrm{s}$ owing to the decrease in $\mathrm{Sn}$ vacancies. ${ }^{8,9}$ There are some reports on the SnEuTe ternally alloy and EuTe/SnTe SL. ${ }^{10-12}$ However, reduction in the carrier concentration has never been reported for this system. Thus, it is considered that the insertion of Eu strongly reduced the number of Sn vacancies in the SnTe layer, leading to the reduction in hole concentration and a drastic increase in hole mobility. Normally, in ternary compounds combined with wide gap materials and IV-VI materials (such as PbEuTe in Table I), the electron mobility is much lower than that of the host IV-VI semiconductor films, because the wide gap elements act as strong scattering centers in the films. This effect also occurs in EuTe/PbTe SLs through the interdiffusion of Eu or interface roughness, and the carrier mobility decreases in the SLs. In the EuTe/SnTe SL, the Eu atoms did not act as significant scattering centers, as indicated by the high hole mobility of the SnEuTe film shown in Table I and the decrease in hole concentration resulting from the decrease in Sn vacancies increased the hole mobility dramatically. As shown in Fig. 2(a), the EuTe layer acts as a very high potential barrier for both electrons and holes in the EuTe/PbTe SL, and the random Eu distribution due to interdiffusion of the constituents results in a strong scattering of the carriers. On the other hand, it is considered that the conduction band bottom in EuTe is located near the narrow band gap of SnTe in the EuTe/SnTe SL. In the case of Fig. 2(b), the Fermi level of SnTe even in the $p$-type sample is situated closer to the conduction band bottom of EuTe in the EuTe/SnTe system, as compared with that of PbTe in the EuTe/PbTe system. Thus, the tunneling probability for holes is higher in the EuTe/SnTe system because they experience a potential barrier up to the conduction band bottom of EuTe, and the Eu atoms do not act as strong scattering centers. There is another possibility that the EuTe/SnTe heterojunction becomes type II where the conduction band bottom of EuTe locates below the valence band top of SnTe. Anyway, the conduction band bottom of 


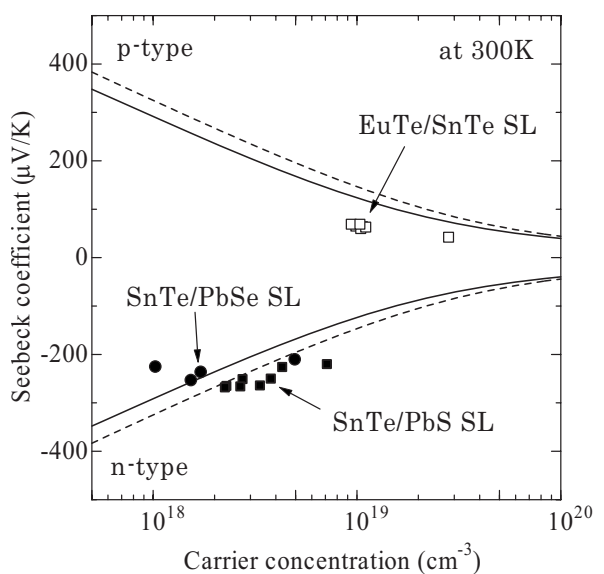

FIG. 4. Seebeck coefficient of SnTe/PbSe, $\mathrm{SnTe} / \mathrm{PbS}$, and EuTe/SnTe SLs. Theoretical curves for PbSe and PbS films are indicated as solid and dashed lines, respectively.

EuTe must locate near the valence band top of SnTe to result the SnEuTe in high hole mobility. It is also considered that the large number of Sn vacancies in the SnTe film forms an impurity band in the valence band, which strongly affects the electrical properties. Thus, the strong decreases in the vacancy concentrations in the SnEuTe film and EuTe/SnTe SL demonstrated the high mobility nature of $p$-type SnTe. Room temperature carrier mobilities of polar semiconductors are strongly affected by optical phonon scattering, with Ge having highest hole mobility of $1900 \mathrm{~cm}^{2} / \mathrm{V} \mathrm{s}$ among semiconductor materials owing to the lack of ionicity. ${ }^{13}$ However, the EuTe/SnTe SL prepared by alternating $26 \mathrm{~nm} \mathrm{SnTe}$ and one monolayer of Eu exhibited a hole mobility of $2720 \mathrm{~cm}^{2} / \mathrm{V} \mathrm{s}$ at room temperature, which is the highest value reported for any $p$-type semiconductor.

Figure 4 shows the dependence of the Seebeck coefficient on the carrier concentration for the $\mathrm{SnTe} / \mathrm{PbSe}, \mathrm{SnTe} /$ $\mathrm{PbS}$, and EuTe/SnTe SLs. SnTe layer thickness for the SnTe/ $\mathrm{PbSe}$ and $\mathrm{SnTe} / \mathrm{PbS}$ SLs are $20-30 \mathrm{~nm}$, and the thicknesses of the $\mathrm{PbSe}$ and $\mathrm{PbS}$ layers are less than 2.5 and $5 \mathrm{~nm}$, respectively, where a clear $n$-type conduction appears. The Seebeck coefficient of the EuTe/SnTe SL was independent of the SnTe thickness. Negative and positive values of the Seebeck coefficients correspond to $n$-type and $p$-type conductions, respectively. Theoretical curves for $\mathrm{PbSe}$ and $\mathrm{PbS}$ films are also drawn as solid and dashed lines, respectively. ${ }^{14}$ The Seebeck coefficients of the $\mathrm{SnTe} / \mathrm{PbSe}$ and $\mathrm{SnTe} / \mathrm{PbS}$ SLs were comparable with the theoretical values for $\mathrm{PbSe}$ and $\mathrm{PbS}$ films. The Seebeck coefficient in the EuTe/SnTe system was smaller than the theoretical values for PbSe and
$\mathrm{PbS}$. We did not calculate the theoretical values for $\mathrm{SnTe}$ and EuTe/SnTe SLs because of the lack of available effective mass data and the complicated band structure around the $L$-points. ${ }^{15}$ It is considered that the small Seebeck coefficient is the result of the complicated band structure at the valence band top, where valence band holes at the $L$-point have a negative effective mass.

In conclusion, $\mathrm{SnTe} / \mathrm{PbSe}, \mathrm{SnTe} / \mathrm{PbS}$, and $\mathrm{EuTe} / \mathrm{PbTe}$ SLs and SnEuTe films were prepared, and their electrical properties were measured at room temperature. The EuTe/ SnTe SL showed very high room temperature hole mobility of up to $2720 \mathrm{~cm}^{2} / \mathrm{V} \mathrm{s}$, which is the highest value among semiconductor materials. The high hole mobility was explained by the reduction in the number of Sn vacancies and the small band offset between conduction band bottom of EuTe and valence band top of SnTe. The SnTe/PbSe and $\mathrm{SnTe} / \mathrm{PbS}$ SLs had a similar band offset to the $\mathrm{SnTe} / \mathrm{PbTe}$ type II SL, and the SLs with thin SnTe layers showed $n$-type conduction. Optical transmission properties of the $\mathrm{SnTe} /$ $\mathrm{PbSe}$ SL indicated semimetallic behavior. The Seebeck coefficients of $\mathrm{SnTe} / \mathrm{PbSe}, \mathrm{SnTe} / \mathrm{PbS}$, and EuTe/SnTe SLs were also measured, and the values for the $\mathrm{SnTe} / \mathrm{PbSe}$ and $\mathrm{SnTe} /$ $\mathrm{PbS}$ SLs were found to be comparable to the theoretical values for $\mathrm{PbSe}$ and $\mathrm{PbS}$ films, respectively.

${ }^{1}$ K. Murase, S. Shimomura, S. Takaoka, A. Ishida, and H. Fujiyasu, Superlattices Microstruct. 1, 177 (1985).

${ }^{2}$ A. Ishida, M. Aoki, and H. Fujiyasu, J. Appl. Phys. 58, 1901 (1985).

${ }^{3}$ S. Takaoka, T. Okumura, K. Murae, A. Ishida, and H. Fujiyasu, Solid State Commun. 58, 637 (1986).

${ }^{4}$ I. Rogacheva, O. N. Nashchekina, A. V. Meriuts, S. G. Lyubchenko, M. S. Dresselhaus, and G. Dresselhaus, Appl. Phys. Lett. 86, 063103 (2005).

${ }^{5}$ A. Ishida, S. Matsuura, M. Mizuno, and H. Fujiyasu, Appl. Phys. Lett. 51, 478 (1987).

${ }^{6}$ A. Ishida, M. Veis, and Y. Inoue, Jpn. J. Appl. Phys., Part 2 46, L281 (2007).

${ }^{7}$ A. Ishida, S. Matsuura, M. Mizuno, Y. Sase, and H. Fujiyasu, J. Appl. Phys. 63, 4572 (1988).

${ }^{8}$ B. B. Houston, R. F. Bis, and E. Gubner, Bull. Am. Phys. Soc. 6, 436 (1961).

${ }^{9}$ H. R. Riedl, J. R. Dixon, and R. B. Schoolar, Phys. Rev. 162, 692 (1967).

${ }^{10}$ J. R. Anderson, M. Gorska, Y. Oka, J. Y. Jen, I. Mogi, and Z. Golacki, Physica B 216, 307 (1996).

${ }^{11}$ A. Y. Ueta, P. H. O. Rappl, H. Closs, P. Motisuke, E. Abramof, V. R. dos Anjos, V. A. Chitta, J. A. Coaquira, N. F. Oliveira, Jr., and G. Bauer, Braz. J. Phys. 34, 672 (2004).

${ }^{12}$ B. Diaz, P. H. O. Rappl, and E. Abramof, J. Cryst. Growth 308, 218 (2007).

${ }^{13}$ S. M. Sze, Physics of Semiconductor Devices, 2nd ed. (Wiley, New York, 1981), Chap. 1.

${ }^{14}$ A. Ishida, T. Yamada, D. Cao, Y. Inoue, M. Veis, and T. Kita, J. Appl. Phys. 106, 023718 (2009).

${ }^{15}$ Y. W. Tsang and M. L. Cohen, Phys. Rev. B 3, 1254 (1971). 